OPEN ACCESS

Edited by:

Jiyu Zhang,

Lanzhou Institute of Husbandry and Pharmaceutical Sciences, Chinese Academy of Agricultural Sciences

(CAAS), China

Reviewed by:

Dandan Hu,

Guangxi University, China

Si-Yang Huang,

Yangzhou University, China

*Correspondence:

Zhengrong Wang

wzrtiger@sina.com

Xinwen Bo

851661263@qq.com

tThese authors have contributed equally to this work

Specialty section:

This article was submitted to

Parasitology

a section of the journal

Frontiers in Veterinary Science

Received: 16 August 2021 Accepted: 05 October 2021 Published: 17 November 2021

Citation:

Xian J, Zhao P, Wang N, Wang W. Zhang $Y$, Meng J, Ma X, Wang Z and Bo X (2021) Molecular

Characterization of a Tetraspanin TSP11 Gene in Echinococcus granulosus and Evaluation Its Immunoprotection in Model Dogs.

Front. Vet. Sci. 8:759283

doi: 10.3389/fvets.2021.759283

\section{Molecular Characterization of a Tetraspanin TSP11 Gene in Echinococcus granulosus and Evaluation Its Immunoprotection in Model Dogs}

\author{
Jinwen Xian ${ }^{1,2 \dagger}$, Pengpeng Zhao ${ }^{1,2+}$, Ning Wang ${ }^{1,2}$, Weiye Wang ${ }^{1,2}$, Yanyan Zhang ${ }^{1}$, \\ Jimeng Meng ${ }^{1}$, Xun $\mathrm{Ma}^{2}$, Zhengrong Wang ${ }^{1 *}$ and Xinwen $\mathrm{Bo}^{1 *}$
}

${ }^{1}$ State Key Laboratory of Sheep Genetic Improvement and Healthy Production/Institute of Animal Husbandry and Veterinary Medicine, Xinjiang Academy of Agricultural and Reclamation Sciences, Shihezi, China, ${ }^{2}$ College of Animal Science and Technology, Shihezi University, Shihezi, China

Cystic echinococcosis (CE) is a cosmopolitan zoonosis caused by the larval stage of Echinococcus granulosus, which affects humans and a wide range of mammalian intermediate hosts. Parasite tetraspanin proteins are crucial for host-parasite interactions, and therefore they may be useful for vaccine development or disease diagnosis. In the present study, the major antigen coding sequence of tetraspanin 11 (Eg-TSP11) from E. granulosus was determined. The results of immunolocalization showed that EgTSP11 was mainly located in the tegument of adult worms and protoscoleces. Western blotting analysis showed that the serum from dogs injected with recombinant Eg-TSP11 (rEg-TSP11) could recognize Eg-TSP11 among natural protoscolex proteins. Moreover, the serum from dogs with E. granulosus infection also recognized rEg-TSP11. Serum indirect enzyme-linked immunosorbent assays demonstrated that IgG levels gradually increased after the first immunization with rEg-TSP11 compared with those in the control group. Furthermore, the serum levels of interleukin 4, interleukin 5, and interferon gamma were significantly altered in the rEg-TSP11 group. Importantly, we found that vaccination with rEg-TSP11 significantly decreased worm burden and inhibited segment development in a dog model of $E$. granulosus infection. Based on these findings, we speculated that $\mathrm{rEg}$-TSP11 might be a potential candidate vaccine antigen against $E$. granulosus infection in dogs.

Keywords: Echinococcus granulosus, cystic echinococcosis, Eg-TSP11, immunogenicity, dog vaccine

\section{INTRODUCTION}

Cystic echinococcosis (CE) is a serious zoonotic parasitic disease caused by Echinococcus granulosus larvae. CE is a major public health concern in developing and developed countries $(1,2)$. In epidemic areas, the incidence of CE is between 1 and 200 per 100,000 $(3,4)$. CE seriously endangers human health and the development of animal husbandry and causes the loss of at least 285,500 disability adjusted life years (DALYs) each year $(5,6)$. The disease is a neglected tropical disease, confirmed by the World Health Organization (WHO) (2). To date, the most successful intermediate 
host vaccine is the EG95 recombinant protein, with a protective efficiency of up to $98 \%$ (7). However, the recombinant EG95 protein had no protective effect on hosts have been infected with E. granulosus and the cysts have been formed. Canids, such as dogs, are the definitive hosts of E. granulosus, which play an important role in the life cycle of E. granulosus. Unfortunately, there is no commercially available definitive-host vaccine, which has seriously hindered the effective control of CE. Therefore, the development of a dog vaccine against E. granulosus is urgently required.

The tetraspanin superfamily (TSP, also known as the transmembrane-4-superfamily, TM4SF) is a hydrophobic plasma membrane-associated protein of 200-350 amino acids. Tetraspanins can be divided into four families, including the cluster of differentiation (CD) family (e.g., CD9, CD81, and CD151), the retinal degeneration slow (RDS) family (e.g., RDS-ROM), the uroplakin family (e.g., UPK1A/1B), and the CD63 family (e.g., CD63 and TSPAN31) (8). The four conserved transmembrane domains of TSPs are called tetraspanin-enriched microdomains (TEMs). TEMs protrude $3-5 \mathrm{~nm}$ from the cell surface to form two extracellular rings, one large and one small (EC1 and EC2). In addition, two short $\mathrm{N}$-and C-terminal cytoplasmic tails are formed in the intracellular region. The large extracellular loop (LEL), which contains 2-6 cysteines, is called the "tetraspanin web" which plays a central role in the interaction of TSPs with several other molecules $(9,10)$.

To date, marked progress has been made in research on tetraspanins as parasite vaccine candidates. Tetraspanin family proteins now occupy an important position in parasite immune interactions, and have been proven to be candidate target proteins for schistosomiasis, Clonorchis sinensis, alveolar echinococcosis, and filariasis (10-13). Tetraspanins are involved in trematode cuticle development, maturation, stabilization, and immune escape (14). The two hydrophilic groups (Sm-TSP-1 and Sm-TSP-2) of the recombinant tetraspanin of Schistosoma mansoni, especially Sm-TSP-2, have been shown to be able to induce a $>40 \%$ immunoprotective effect against $S$. mansoni infection (15). The TSP1 gene of E. granulosus was cloned and expressed for the first time in 2015 and it was demonstrated that TSP1 could stimulate a marked Th1 type immune response in a mouse model. This means that TSP1 may be a possible target in the treatment, prevention, and control of echinococcosis (8). Tetraspanins play important roles in the study of the life cycle of parasites and in mediating parasite signal transduction, immune escape, and other important biological processes. As cell surface molecules, tetraspanins play important roles as bridges in parasite cell signaling pathways and thus represent potential therapeutic targets (9). The present study aimed to characterize the biological characteristics of TSP11 in E. granulosus. In addition, the immunoprotective effect of recombinant tetraspanin $11 \mathrm{Eg}$ TSP11 (rEg-TSP11) was analyzed by evaluating changes in the levels of IgG, and the cytokines Th1 and Th2 in model dogs. Furthermore, we analyzed the worm burden reduction rate and the inhibition of segment development in rEg-TSP11vaccinated dogs. The results of the present study provide basic immunogenicity data for rEg-TSP11 and pave the way for the development of anti-E. granulosus vaccines in dogs.

\section{MATERIALS AND METHODS}

\section{Parasites}

Naturally infected sheep livers, obtained from an abattoir in Urumqi, Xinjiang Province, China, were the source of hydatid cysts. Cyst fertility was checked using light microscopy to determine the presence of protoscoleces (PSCs) within the cysts. PSCs were isolated and treated according to previously described methods (16). PSCs $(n=2,000)$ were grown in $1 \mathrm{~mL}$ Roswell Park Memorial Institute (RPMI) 1640 medium containing $100 \mu \mathrm{g} / \mathrm{mL}$ streptomycin and $100 \mathrm{U} / \mathrm{mL}$ penicillin (Sigma-Aldrich, St. Louis, MO USA) and $10 \%$ bovine serum albumin (BSA; Hyclone, Logan, UT, USA). At 28 days after artificial PSC infection in an 8-month-old dog, adult worms were obtained.

\section{Bioinformatic Analysis}

The cDNA sequence of Eg-TSP11 (XP_024352489.1) was downloaded from the NCBI database. The physicochemical parameters of the encoded proteins were analyzed using ProtParam tools on the ExPASY website (https://web.expasy. org/protparam/). SignalP 4.1 (http://www.cbs.dtu.dk/services/ SignalP/) was used to check for the presence of a signal peptide, and Novopro tools were used to analyze the transmembrane regions (https://www.novopro.cn/tools/tmhmm.html). SWISSMODEL (http://swissmodel.expas y.org/) was used to model the tertiary (three-dimensional) structures. The B cell epitopes of EgTSP11 were predicted using the IEDB online server (http://tools. immuneepitope.org/main/). MEGA software (version 5.05) used the neighbor-joining $(\mathrm{NJ})$ method to construct phylogenetic trees of aligned proteins (17).

\section{Expression and Purification of the Eg-TSP11 LEL Region}

An RNA-prep Pure Tissue Kit (Nanjing Vazyme Biotech, Nanjing, China) was used to extract total RNA from the PSCs. First-strand cDNA was synthesized from total RNA using a reverse transcription system kit (Nanjing Vazyme Biotech). PCR was then used to amplify the LEL coding sequence from the cDNA using a sense primer (5'- CGC GGA TCC ATG TTT CCA GCA CCG CTT CAA G-3') comprising a BamHI site (underlined) and an antisense primer (5'-CCG CTC GAG TCA TTC ATA GTT TTT CAA GGA G-3') comprising a XhoL I site (underlined). The PCR amplicons were ligated into the pET32a $(+)$ plasmid (Novagen, Darmstadt, Germany) and transformed into Escherichia coli BL21 (DE3) cells (Tiangen, Beijing, China). Isopropyl-1-thio- $\beta$-D-galactopyranoside (1 mM; IPTG) was used to induce expression from the plasmid for $6 \mathrm{~h}$ at $37^{\circ} \mathrm{C}$. Inclusion bodies were obtained from the E. coli cells, suspended in lysis buffer containing $8 \mathrm{M}$ urea, and incubated for $2.5 \mathrm{~h}$ on ice to completely solubilize the recombinant protein. $\mathrm{Ni}^{2+}$ affinity chromatography with a His-affinity resin column (BioRad, Hercules, CA, United States) was used to purify the Histagged rEg-TSP11 protein, which was subjected to $10 \%$ sodium dodecyl sulfate-polyacrylamide gel electrophoresis (SDS-PAGE). A NanoDrop 2000c (Bio-Rad) instrument was used to determine protein concentration. 


\section{Western Blotting}

SDS-PAGE (10\%) was used to separate rEg-TSP11 and total proteins in extracts from PSCs. The separated proteins were then electrotransferred onto nitrocellulose membranes. Membranes were blocked with $5 \%(\mathrm{w} / \mathrm{v})$ skim milk for $2 \mathrm{~h}$ at $37^{\circ} \mathrm{C}$. The membranes were then incubated with E. granulosus positive or negative dog sera, anti rEg-TSP11 dog sera, or pre-immunized mouse sera (1:100 v/v dilutions) at $4^{\circ} \mathrm{C}$ overnight. The next day, the membranes were washed and incubated with horseradish peroxidase (HRP)-conjugated rabbit anti-dog IgG or sheep antimouse IgG (1:5,000 v/v dilution) for $2 \mathrm{~h}$. Actin protein of $E$. granulosus was used as an internal control. The membranes were incubated with Pierce ECL western blotting substrate (Thermo Fisher Scientific, Waltham, MA, USA) and then exposed to $\mathrm{X}$-ray films.

\section{Quantitative Real-Time Reverse Transcription PCR}

Quantitative real-time reverse transcription PCR (qRT-PCR) was used to examine the expression profiles of Eg-TSP11 in 28-day strobilated worms and PSCs. Total RNA was isolated from 28-day strobilated worms and PSCs, and cDNA was synthesized from total RNA. The cDNA was then used as a template for $\mathrm{qPCR}$ using the TSP11 primers: 5' - GAA GAT AAT GGC TGG GGT GC- $3^{\prime}$ and $5^{\prime}$-GTT GTG TGC CCC ATT TGT GA-3'. Actin gene expression was used as an internal control for normalization. Primers for amplification of E. granulosus actin were $5^{\prime}$-GAG TCA TGT AGG CCA CG- $3^{\prime}$ and $5^{\prime}$-AGA TGG AGG TGG GGA TAG G-3'. The $2^{-\Delta \Delta C T}$ method was used to analyze the data (18).

\section{Immunolocalization}

To identify the location of TSP11 at different developmental stages, adult worms and fresh PSCs were fixed overnight using $4 \%$ paraformaldehyde hydrophosphate buffer, permeabilized for $30 \mathrm{~min}$ using $1 \%$ Triton $\mathrm{X}-100$, and incubated in $0.01 \%$ Triton $\mathrm{X}-100$ at $4^{\circ} \mathrm{C}$ for $1 \mathrm{~h}$. They were then washed three times with $0.01 \times$ phosphate-buffered saline (PBS) and blocked with $5 \%$ (w/v) skim milk for $2 \mathrm{~h}$ at $37^{\circ} \mathrm{C}$. Next, the fixed adult worms and PSCs were incubated with anti-rEg-TSP11 dog IgG (1:100 v/v dilution in PBST) at $4^{\circ} \mathrm{C}$ overnight. After washing, the sections were incubated with phycoerythrin (PE)-conjugated goat anti$\operatorname{dog} \operatorname{IgG}(\mathrm{H}+\mathrm{L})(1: 1,000 \mathrm{v} / \mathrm{v}$ dilutions in PBST) for $2 \mathrm{~h}$ in the dark at room temperature $\left(25^{\circ} \mathrm{C}\right)$. The sections were washed four times with PBST and then examined under a fluorescence microscope (Leica, Wetzlar, Germany). The negative control was comprised of antibodies from the pre-immunized mice.

\section{Vaccination and $E$. granulosus Challenge}

The vaccination experiment included nine Beagles. Group 1 was comprised of three dogs that were vaccinated with rEgTSP11 mixed with the saponin adjuvant Quil A; Group II was comprised of three dogs that were vaccinated with Quil A only; and Group III was comprised of three dogs that were vaccinated with PBS (control group). Each $350 \mu \mathrm{L}$ dose of vaccine included $200 \mu \mathrm{g}$ of soluble rEg-TSP11 and $100 \mu \mathrm{g}$ of Quil A (Sigma) in PBS. Before vaccination, the mixture was stirred overnight at $4{ }^{\circ} \mathrm{C}$. All experiments beagle dogs were immunized through subcutaneous injection in the neck and all the dogs were immunized with the same dose four times, at a 14 days interval. Seven days after the last booster vaccination, all nine dogs were challenged orally with 100,000 E. granulosus PSCs. Finally, at 28 days after infection (before eggs appeared), all nine dogs were euthanized and necropsied to collect and count worms as previously described $(19,20)$. Thirty worms were chosen randomly from each experimental group and the sizes of developed ( $\geq 4$ segments) vs. underdeveloped ( $\leq 3$ segments) worms were determined (21).

\section{Indirect Enzyme-Linked Immunosorbent Assay (ELISA)}

The standard checkerboard titration procedure was used to assess the optimal concentrations of the rEg-TSP11 antigen and serum. Carbonate buffer (0.1 M, pH 9.6) was used to dilute the purified $\mathrm{rEg}$-TSP11 protein to $5 \mu \mathrm{g} / \mathrm{mL}$, which was used as the antigen in the ELISA test. The diluted antigen solution was used to coat ELISA plates at $4^{\circ} \mathrm{C}$ overnight. The next day, the plate was washed with PBS-Tween-20 (PBST) and then incubated with 5\% skim milk at $37^{\circ} \mathrm{C}$ for $2 \mathrm{~h}$. After thorough washing, $100 \mu \mathrm{L}$ of serum samples (2-fold dilutions, 1:80) in PBST was added to each well and incubated for $1.5 \mathrm{~h}$ at $37^{\circ} \mathrm{C}$. After washing, HRP-labeled rabbit anti-dog IgG (1:3,000 dilution; Solarbio, Beijing, China) was added to the plates and incubated for $1.5 \mathrm{~h}$ at $37^{\circ} \mathrm{C}$. After washing, the substrate, 3, 3', 5, 5' -tetramethylbenzidine (TMB) (Tiangen, Beijing, China) was added to the wells and incubated for $1.5 \mathrm{~h}$ at $37^{\circ} \mathrm{C}$. Finally, $1 \mathrm{M} \mathrm{H}_{2} \mathrm{SO}_{4}(100 \mu \mathrm{L})$ was added to stop the development of color, and the optical density was measured at $450 \mathrm{~nm}(\mathrm{OD} 450)$.

\section{Cytokine Detection}

Five main cytokines in dogs with E. granulosus infection, including interferon gamma (IFN- $\gamma$ ), interleukin (IL)-6, IL-5, IL-4, and IL-1 were determined using an ELISA kit (Janglaibio, Shanghai, China). The required strip was removed from the aluminum foil bag after being left for $60 \mathrm{~min}$ at room temperature $\left(25^{\circ} \mathrm{C}\right)$. The standard sample well, blank well, and sample well were set, and $50 \mu \mathrm{L}$ of standard product at different concentrations was added. Then, HRP-labeled antibody $(100 \mu \mathrm{L})$ was added to each well, which was sealed using a sealing film and incubated for $1 \mathrm{~h}$ at $37^{\circ} \mathrm{C}$. The liquid was discarded, and the plate was patted dry using absorbent paper. Next, $350 \mu \mathrm{L}$ of detergent was added to each well and incubated for $1 \mathrm{~min}$, after which the detergent was shaken and the plate was patted dry using absorbent paper. The detergent wash step was repeated five times. Then, $50 \mu \mathrm{L}$ of substrate A and $50 \mu \mathrm{L}$ of substrate B were added to each well and incubated for $15 \mathrm{~min}$ at $37^{\circ} \mathrm{C}$. Finally, 50 $\mu \mathrm{L}$ of termination solution was added to each well, and within $15 \mathrm{~min}$, the OD value of each well was measured at a wavelength of $450 \mathrm{~nm}$.

\section{Data Analysis}

Statistical analyses were performed using SPSS software (version 22.0; IBM Corp, Armonk, NY, USA). All data analyses and graphs were performed using GraphPad Prism 6.0 software package (GraphPad Software Inc., San Diego, CA, USA). Statistical 
significance was set at $P<0.05$. All experiments were repeated a minimum of three separate times.

\section{RESULTS}

\section{Bioinformatic Analysis}

The Eg-TSP11 cDNA sequence was comprised of 765 nucleotides, which encoded a putative protein of 254 amino acids (aa). TSP11 had a predicted pI of 8.91, a predicted mass of 27 $\mathrm{kDa}$, three potential N-terminal glycosylation sites, five potential protein kinase phosphorylation sites, and one predicted tyrosine kinase phosphorylation site (Figure 1), including the three typical transmembrane regions (17-39, 59-81, and 94-116). TSP11 contains a NET-5-LIKE-LEL (the largest extracellular loop) located at amino acids 116-120 aa. TSP11 comprises seven predicted B cell linear epitopes (ep1: 48-55 aa; ep2: 123-123 aa; ep3: 143-143 aa; ep4: 162-170 aa; ep5: 186-189 aa; ep6: 210-219 aa; and ep7: 227-240 aa). The three-dimensional structure of TSP11 (judged by structural modeling) revealed multiple helices and folding regions, and its three-dimensional

\section{MAFRLRPFVLRIFIHAVNVILGVTNLYLFIVKFFGVFILSLSIFTSLNKSNTPEILGNYL}

61 FSGGVYSALFCSIFLIFLPIWGSIALKRYSRLMLILYVIGIATLIIVTFCAGTSLIVFPA

121 PLQAAVKLEMNKTLYHEYGKRGFITDSWDFVQSFLRCCAVEDNGWGAYNGSWWDLSVNAY

181 FYSVDSRLPETSLFYKRVPKSCCLTLVDPLTGWLPTDQYQNVLQCQNWQYGPPRFTNGAHN

\section{DALYYRVSSLKNYE}

FIGURE 1 | The deduced amino acids sequence of TSP11. Underlined: five protein kinase phosphorylation sites; border marked on frame: three potential N-terminal glycosylation sites; shaded: one tyrosine kinase phosphorylation site.

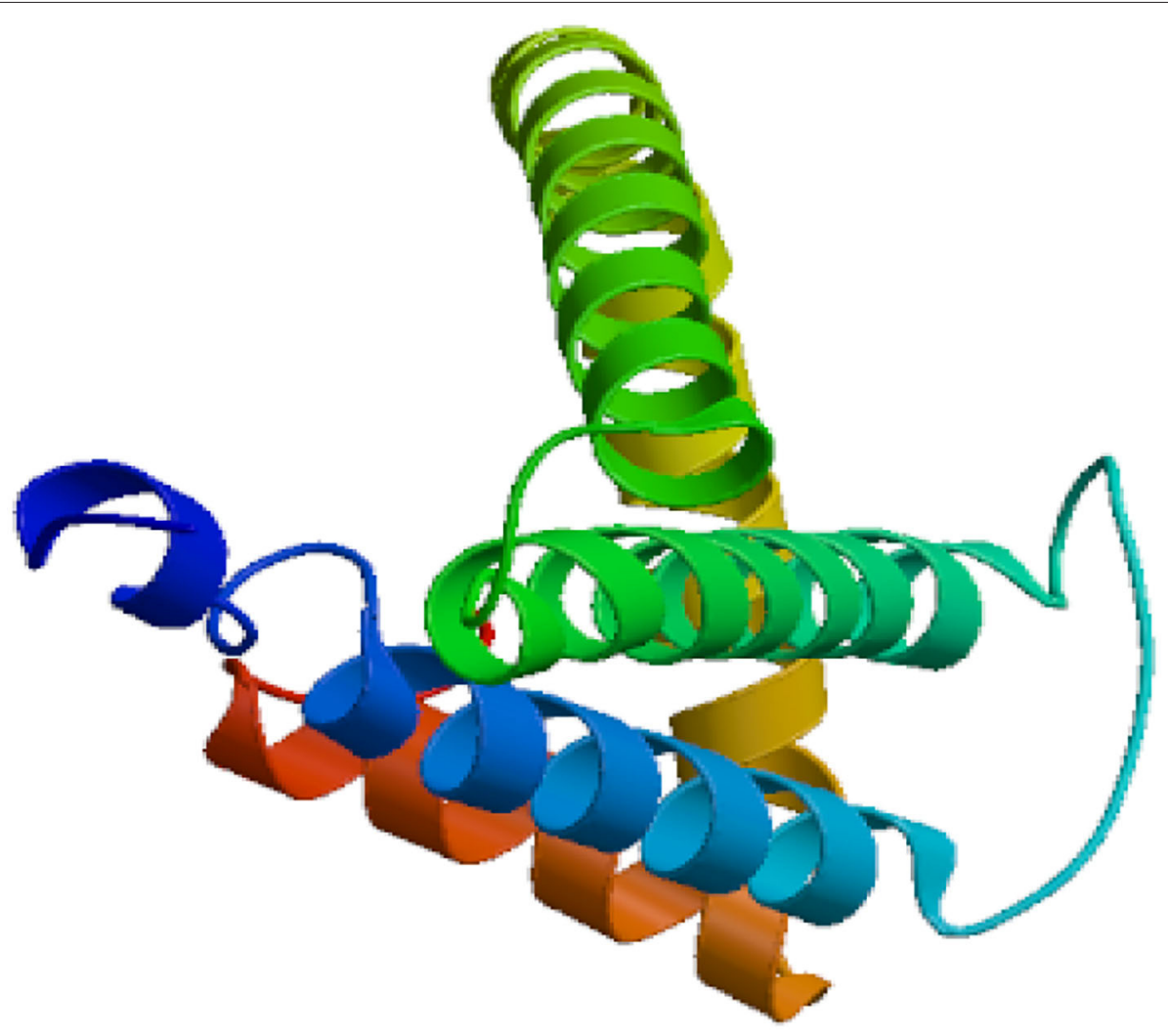

FIGURE 2 | Predicted secondary and three-dimensional structure of the Eg-TSP11 protein. 


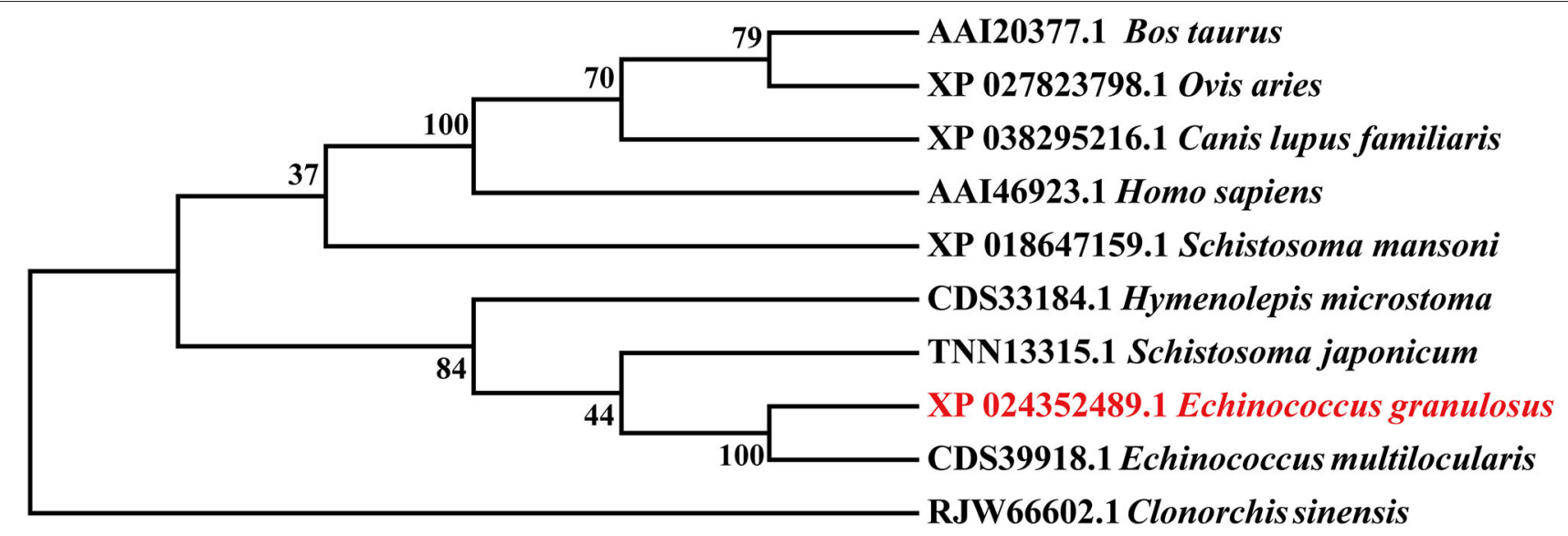

FIGURE 3 | Neighbor Joining phylogenetic tree constructed using Eg-TSP11 and TSP proteins from other species.

A

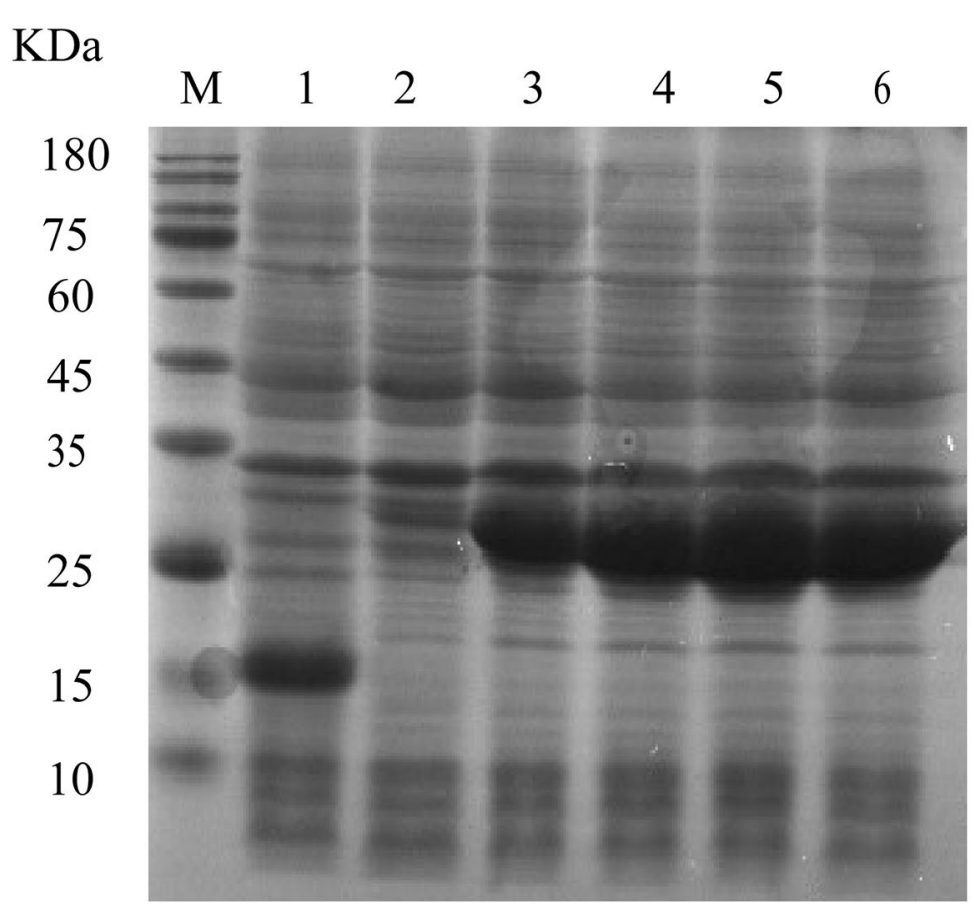

B

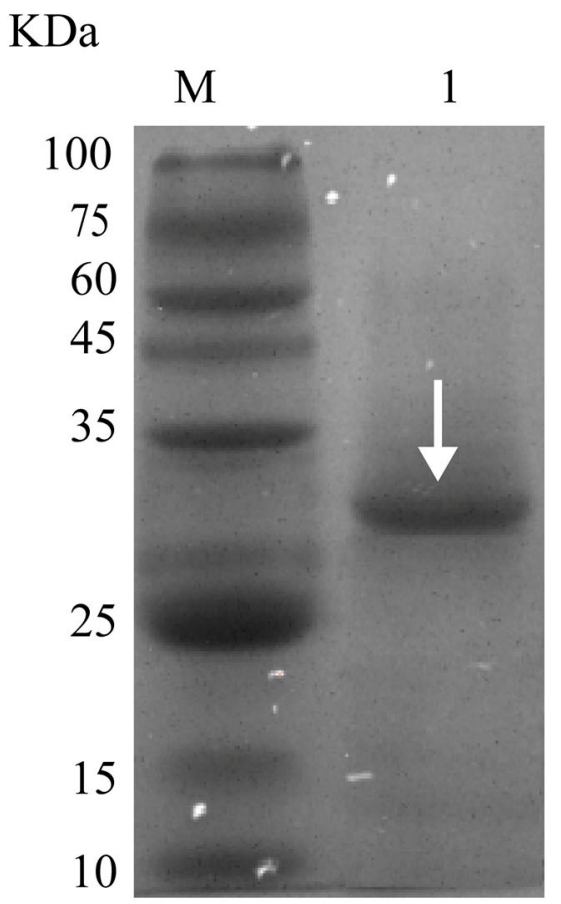

FIGURE 4 | rEg-TSP11 expression and purification. (A) Lane M: Protein molecular weight markers; Lane 1, The recombinant plasmid was transferred into E. coli BL21 (DE3) followed by IPTG induction of pET-32a (+) empty vector for $6 \mathrm{~h}$. Lane 2-6: Recombinant plasmids were transferred into E. coli BL21 (DE3) followed by IPTG induction of pET32a-TSP11 for 0, 2, 4, 6, and 8 h. (B) Lane M: Protein molecular weight markers. Lane 1: protein sample following purification of the His-tagged rTSP11 protein using an $\mathrm{Ni}^{2+}$ column.

structure was mainly comprised of irregular coils (Figure 2). In the phylogenetic analysis, Eg-TSP11 was similar to TSP proteins from Echinococcus multilocularis (Figure 3).

\section{Expression, Purification, and Recognition of} rEg-TSP11

The cDNA encoding the LEL of Eg-TSP11 was PCR-amplified from PSCs, and the recombinant rEg-TSP11 was expressed successfully. The purified rEg-TSP11 protein, which included a His-tag, showed a single band close to the predicted size of 32 $\mathrm{kDa}$ on $10 \%$ SDS-PAGE (Figure 4).

\section{Western Blotting}

Immunoblotting using sera from dogs experimentally infected with E. granulosus showed a single $\sim 32 \mathrm{kDa}$ band. The native Eg-TSP11 protein from PSC extract could be recognized using 
A
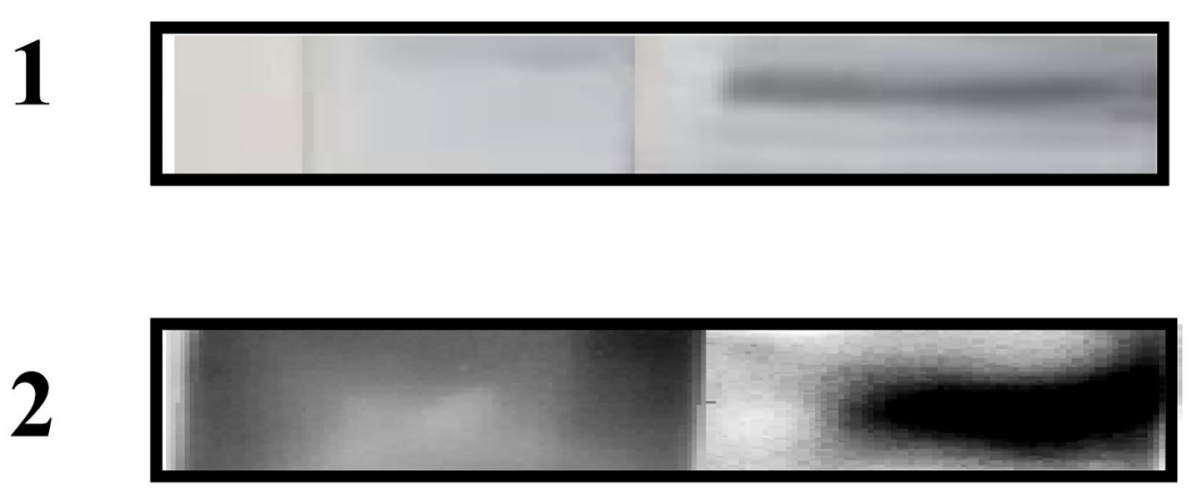

3
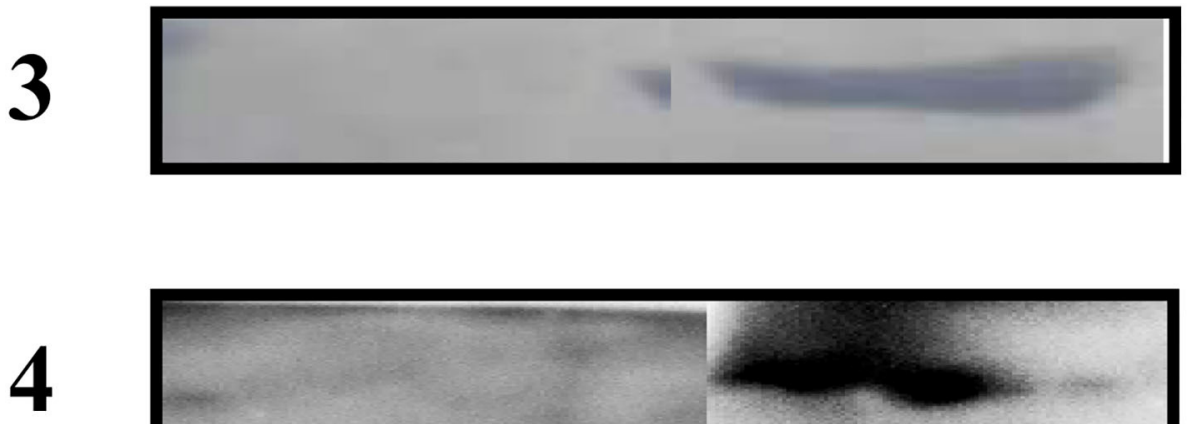

B

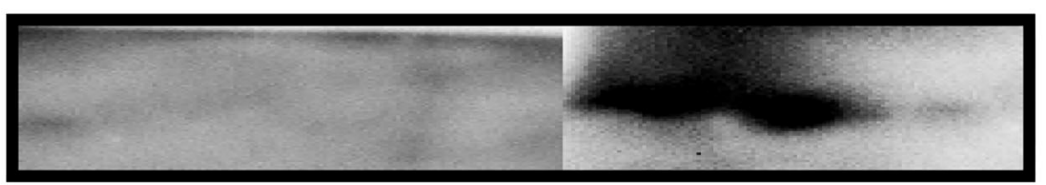

FIGURE 5 | Western blotting analysis. (A) Lane A1: total protein extracts of PSCs probed with pre-immunized dog sera; Lane A2: total protein extracts of PSCs probed with pre-immunized dog sera; Lane A3: non-infected dog sera was used to probe purified rEg-TSP11; Lane A4: non-infected dog sera was used to probe purified rEg-actin. (B) Lane B1: total protein extracts of PSCs probed using anti-rEg-TSP11 dog sera; Lane B2: total protein extracts of PSCs probed using anti-rEg-actin dog sera; Lane B3: purified rEg-TSP11 probed with the serum of E. granulosus infected dog; Lane B4: purified rEg-actin probed with the serum of E. granulosus infected dog.

anti-rEg-TSP11 dog IgG, with an apparent molecular mass of $\sim 27 \mathrm{kDa}$, as expected. As anticipated, when reacted with preimmunized dog serum or non-infected dog serum, no signal was observed with rEg-TSP11 (Figure 5).

\section{qRT-PCR Analysis of Eg-TSP11 Expression}

The relative transcription of Eg-TSP11 was assessed using qRTPCR. Eg-TSP11 mRNA was detected in E. granulosus adult and protoscolex stages, with a significantly higher level in the PSC stage $(P>0.05$; Figure 6).

\section{Eg-TSP11 Localization During Different Life Stages of E. granulosus}

The localization of native Eg-TSP11 in PSCs and adult worms was determined by immunofluorescence using specific polyclonal antibodies against rEg-TSP11. In PSCs, the fluorescence signals were mainly localized in the tegument tissues and hook, while weak signals were detected in the parenchymal region (Figure 7A). In adult worms, weak signals were detected in tegument tissue (Figure 7B). No fluorescence signals were detected in the negative control samples.

\section{Indirect ELISA}

Indirect ELISA was used for a preliminary assessment of serum IgG changes after vaccination with $\mathrm{rEg}$-TSP11. At $0,14,28$, and 42 days after the first vaccination, sera from nine immunized dogs were tested. In the dogs immunized with rEg-TSP11, the IgG level increased significantly at 14,28 , and 42 days after immunization $(14 \mathrm{~d} t=11.35 P=0.0003,28 \mathrm{~d} t=7.700, P=0.00151,42 \mathrm{~d} t=$ $14.89, P=0.0001)$ and at 28 days post-challenge compared with the PBS control group $(t=4.729, P=0.0091)$. IgG levels peaked 42 days post-vaccination (Figure 8).

\section{Response of Canine Cytokines Induced by rEg-TSP11}

ELISA was used to detect cytokines IFN- $\gamma$, IL-6, IL-5, and IL4 at $0,14,28$, and 42 days after immunization and at 28 days post-PSC challenge (Figure 9). Compared with that in the PBS 


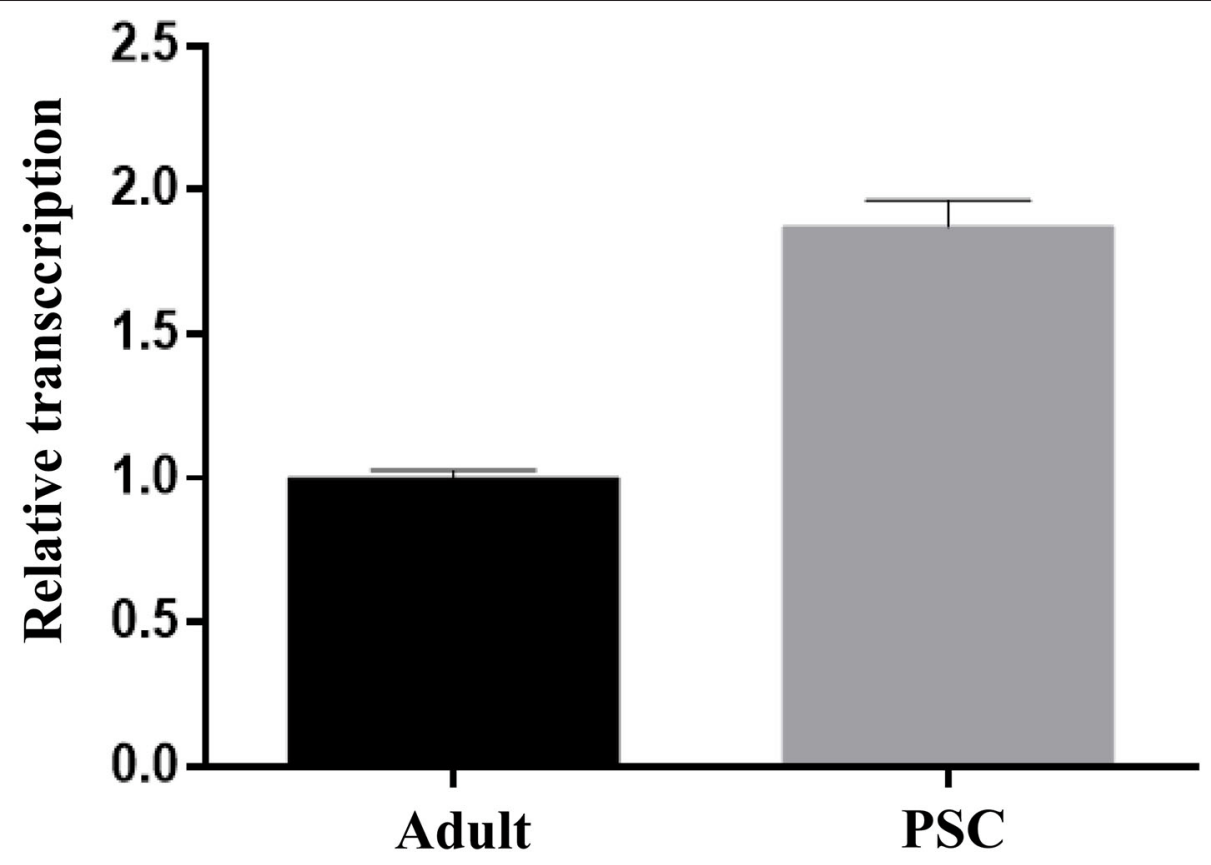

Developemental stages

FIGURE 6 | Expression analysis of Eg-TSP11 mRNA in different developmental stages of E. granulosus.

control group, the serum IL-4 level increased significantly in the rEg-TSP11 vaccinated dogs at 42 days after immunization $(14 \mathrm{~d}$ $t=0.09306 P=0.9303,28 \mathrm{~d} t=2.129, P=0.1003,42 \mathrm{~d} t=$ 5.786, $P=0.0045)$; however, there was no significant change at day 28 post-PSC challenge ( $t=0.3628, P=0.7351$ ) (Figure 9A). Moreover, the serum IL-5 level increased significantly at 42 days after immunization (14 d $t=0.3599 P=0.7372,28 \mathrm{~d} t=$ $0.3042, P=0.7761,42 \mathrm{~d} t=11.08, P=0.0004)$ and 28 days post-PSC challenge $(t=3.104, P=0.0361)$ in the $\mathrm{rEg}$-TSP11 vaccinated dogs (Figure 9B). Furthermore, the serum IFN- $\gamma$ level increased significantly at 28 days and 42 days after immunization (14 d $t=2.592 P=0.0605,28 \mathrm{~d} t=6.200, P=0.0034,42$ $\mathrm{d} t=2.803, P=0.0487)$. There were no significant changes at day 28 post-PSC challenge $(t=0.4121, P=0.7014)$ in the rEg-TSP11 vaccinated dogs (Figure 9C). In addition, there were no significant differences in serum IL-6 (Figure 9D) and IL-1 (Figure 9E) levels in rEg-TSP11 vaccinated dogs in the different groups.

\section{Vaccine Efficacy of rEg-TSP11 Combined With Quil A} Vaccination using rEg-TSP11 mixed with Quil A resulted in a $76.80 \%$ reduction in the number of worms compared to that in the PBS control group (Table 1). This suggested that vaccination with rEg-TSP11 mixed with Quil A induced good protective efficacy (inhibition of worm growth) in Beagles against E. granulosus infection 28 days after PSC challenge.

\section{Worm Development in rEg-TSP11 Vaccinated Dogs}

The results showed that in the rEg-TSP11-vaccinated group, the development and maturation of the worms was inhibited by $77.04 \%$. Thus, vaccination with rEg-TSP11 induced a significant protective effect, as indicated by the inhibition of worm growth (Table 2).

\section{DISCUSSION}

Tetraspanins are widely distributed in eukaryotes and participate in many cellular biological processes (22). They play a significant downregulatory role, especially in immune interactions with host molecules, including the major histocompatibility complex (MHC) $(23,24)$. This immune interaction is vital in the process of parasite immune evasion. The downregulation of the host immune response by tetraspanin allows the parasite to hide its non-self-state and evade detection by the host, leading to successful host infection. Therefore, targeting tetraspanins using a variety of methods, including RNA interference or monoclonal antibodies, could lead to the development of antiparasite vaccines. Certain members of the tetraspanin family have been targeted in schistosomiasis (25-27), Clonorchiasis sinensis (28), and alveolar echinococcosis $(29,30)$, and as candidate vaccines for filariasis (31). However, there are few reports on the TSPs of E. granulosus. In this study, molecular cloning, prokaryotic expression, western blotting, and fluorescence immunolocalization were carried out. The immune protective effect of the rEg-TSP11 in dogs was evaluated by assessing 


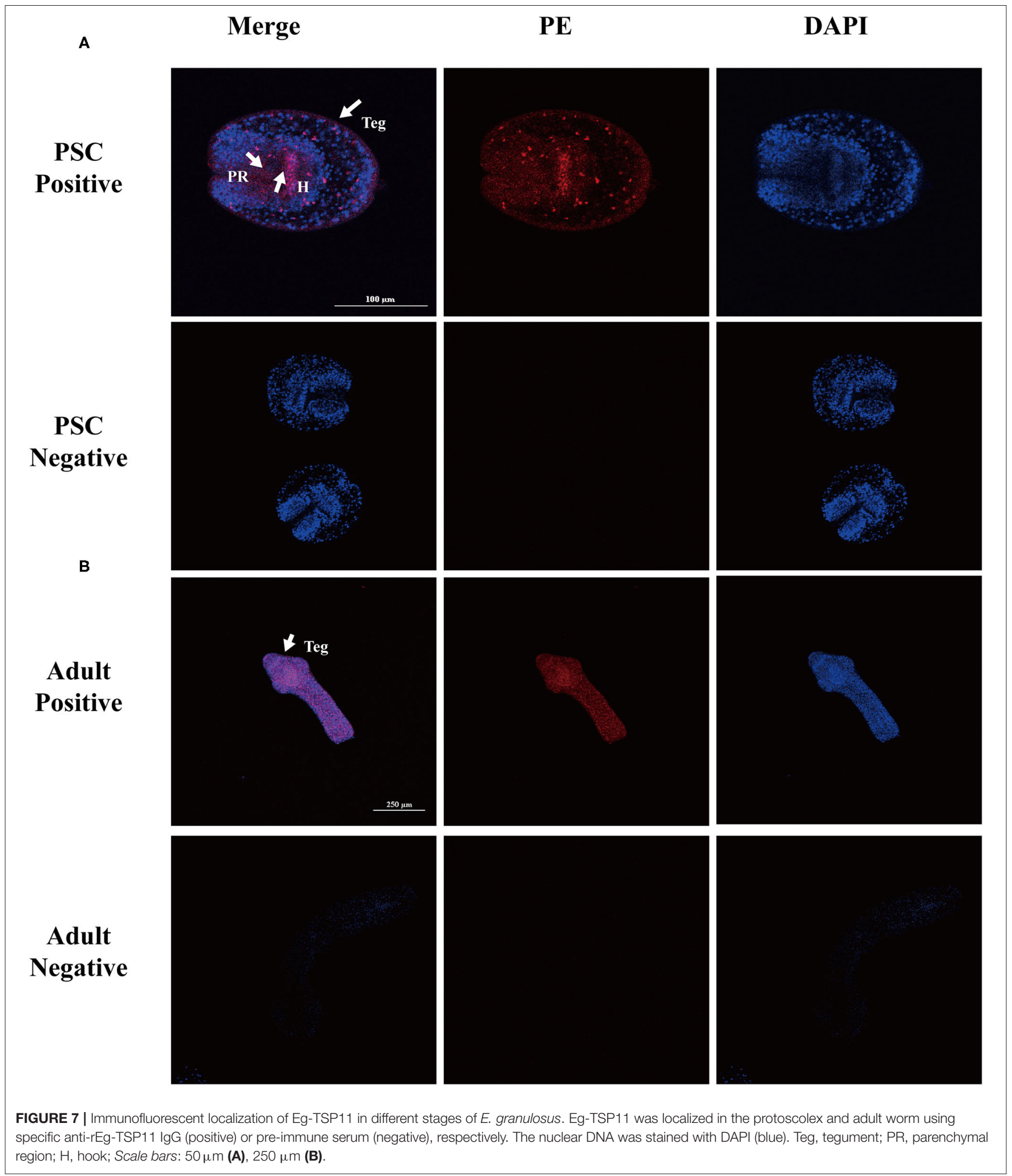

the changes in antibody levels, the worm reduction rate, the inhibition of worm segmentation, and Th1/Th2 cytokine levels. These results provide the basis for determining the antigenic potential of rEg-TSP11.
The results of bioinformatic analysis showed that TSP11 has five protein kinase phosphorylation sites and one tyrosine kinase phosphorylation site, indicating that phosphorylation of TSP11 plays a pivotal role in E. granulosus growth and development 


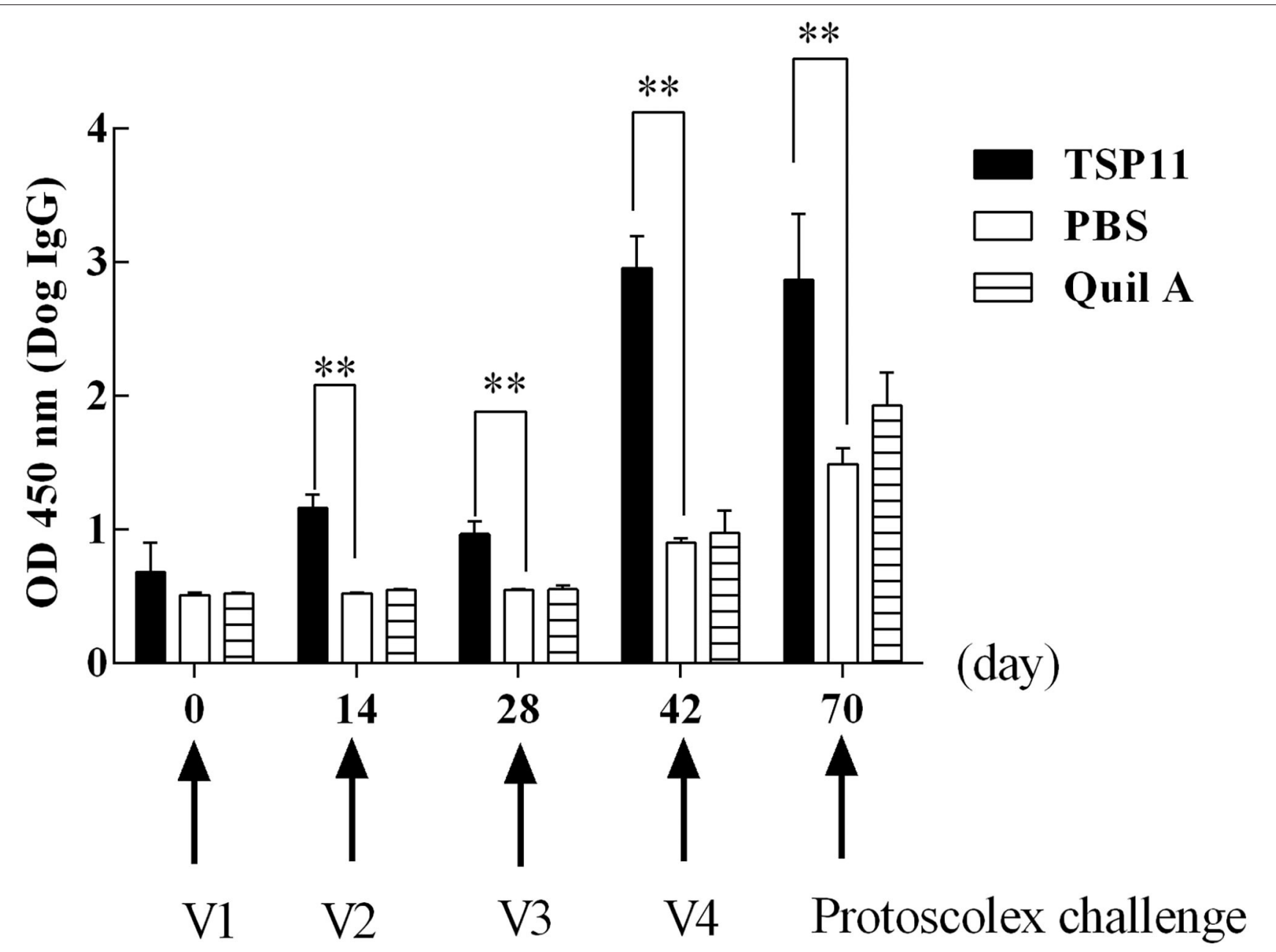

FIGURE 8 | Changes in antibody lgG levels in dogs vaccinated with rTSP11+Quil A, Quil A alone, or PBS. ${ }^{\star} P<0.05 ;{ }^{* *} P<0.01$.

(32). Specific epitopes determine the specificity of antigens (33). Therefore, epitope prediction is particularly important in the study of protein structure and function, and for the diagnosis of diseases and the design of vaccines (34). The present study found that there were seven potential B-cell epitopes in EgTSP11, among which the random coil regions were 227-240 (amino acid sequence: WQYGPPRFTNGAHN) and 210-219 (amino acid sequence: LTGWPTDQYQ). The results showed that these two regions had strong antigenicity, and most of the epitopes were located in the extracellular loop of tetraspanin LEL, which has a highly conserved cysteine sequence. Studies have shown that the LELs of some TSPs have good immunogenicity and reactivity and are potential vaccine candidate antigens (8); therefore, in the present study, we cloned and expressed the LEL of Eg-TSP11. This analysis indicated that Eg-TSP11 has good antigenic potential and provides a theoretical basis for Eg-TSP11 as a candidate antigen for the development of a vaccine against $E$. granulosus infection. Transcriptional analysis indicated that Eg-TSP11 is expressed in both adult worms and the protoscolex, suggesting that Eg-TSP11 might play a role in the parasite life cycle. Moreover, we found that Eg-TSP11 was mainly expressed in the epidermis of adult worms and protoscolex. Interestingly, we also found that the serum from dogs with E. granulosus infection also recognized rEg-TSP11. In platyhelminths, the tetraspanin families are expanded and are likely to be components of the host-pathogen interface (35). Studies have shown that the tetraspanins could be part of extracellular vesicles that are released by helminths within hosts (36), that they bind to the Fc domain of host antibodies (37), and that they are highly immunogenic (38). Based on these findings, we speculated that Eg-TSP11 might be a component of the hostpathogen interface and could be part of the extracellular vesicles released by E. granulosus within hosts.

Quil A, as a saponin adjuvant, is widely used in veterinary vaccines and human and veterinary immunology. Saponins can induce strong adjuvant responses to $\mathrm{T}$ cell-dependent antigens (39). Saponins can also induce a strong cytotoxic $\mathrm{CD}^{+}$lymphocyte reaction and enhance the response to mucosal antigens (40), and when combined with cholesterol and phospholipids, immune stimulating complexes are formed 

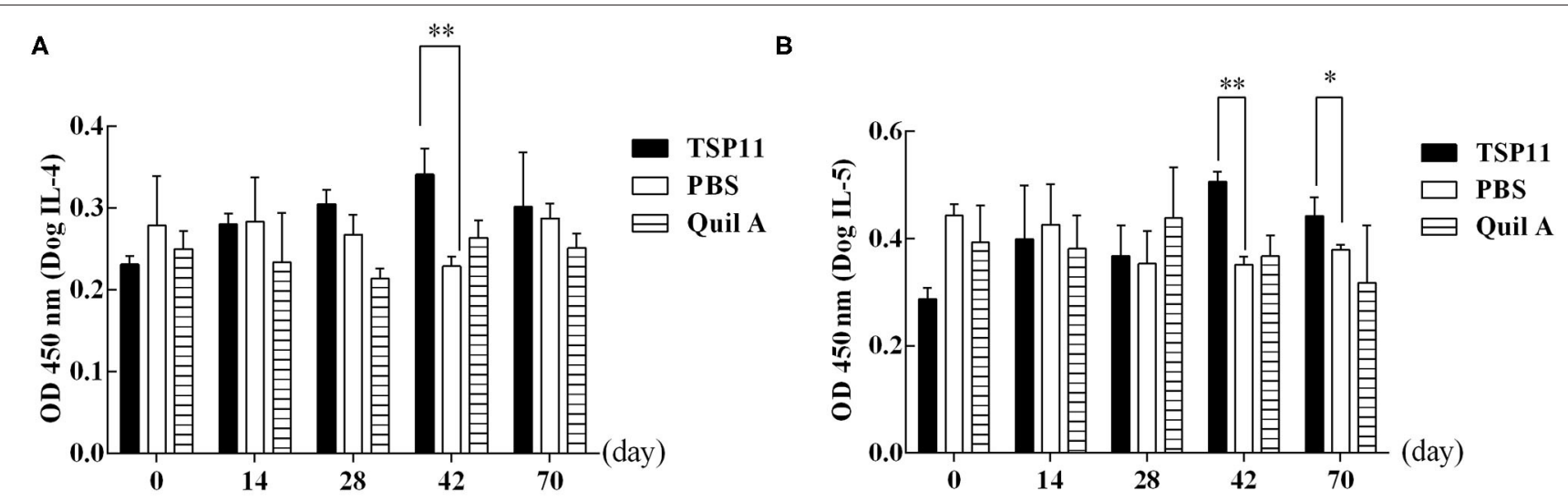

c

D
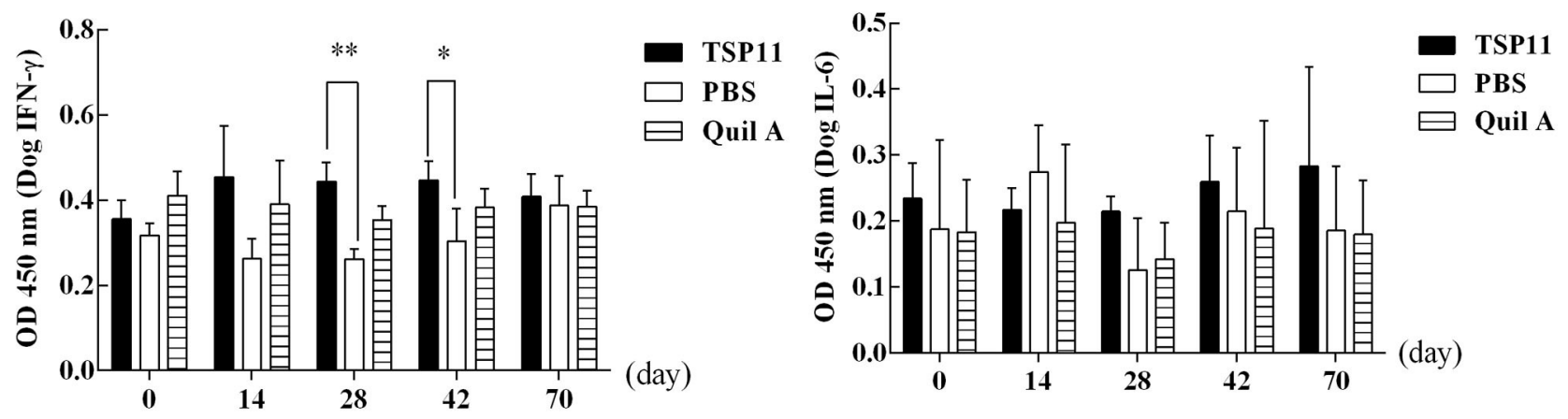

E

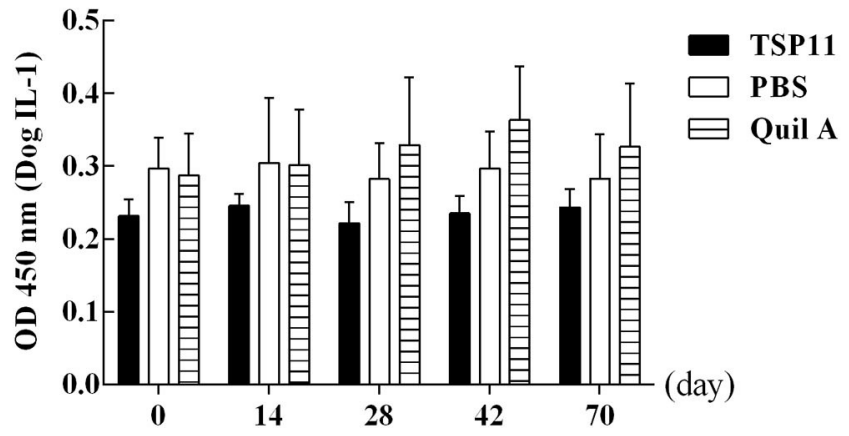

FIGURE 9 | ELISA detection of the levels of different cytokines in dogs vaccinated with rTSP11+Quil A, Quil A alone, or PBS. (A) IL-4, (B) IL-5, (C) IFN- $\gamma$, (D) IL-6, E: IL-1. ${ }^{\star} P<0.05 ;{ }^{* \star} P<0.01$.

(41). The Quil A adjuvant can activate cellular and humoral immune responses against a variety of viruses, bacteria, parasites, and tumor antigens $(41,42)$. Hence, in this study, we used rEg-TSP11 mixed with Quil A to induce a more effective immune response. Indirect ELISA was used to detect changes in IgG levels in dog serum. The results showed that rEgTSP11 could significantly stimulate the body to produce specific antibodies. 
TABLE 1 | Number of Worms in vaccinated dogs after PSC challenge.

\begin{tabular}{|c|c|c|c|}
\hline Group (protein) & Dog ID number & Number of worms & Reduction $\%^{a}$ \\
\hline \multirow[t]{3}{*}{ PBS } & 1 & 13,200 & \\
\hline & 2 & 4,800 & \\
\hline & 3 & 5,700 & \\
\hline Average & & 7,900 & - \\
\hline \multirow[t]{3}{*}{ Quil A } & 4 & 28,200 & \\
\hline & 5 & 4,200 & \\
\hline & 6 & 4,800 & \\
\hline Average & & 12,400 & \\
\hline \multirow[t]{3}{*}{ Eg-TSP11 } & 7 & 1,200 & \\
\hline & 8 & 1,400 & \\
\hline & 9 & 2,900 & \\
\hline Average & & 1,833 & 76.80 \\
\hline$P$-value ${ }^{b}$ & & & 0.100 \\
\hline
\end{tabular}

Echinococcosis is a zoonotic parasitic disease that is also an immune imbalance disease (42). E. granulosus infection initiates long-term interactions with the host. In this interaction, the existence of Th1/Th2 type immune responses plays a crucial role. When the worm changes the immune balance of Th1 and Th2 in the host's own system via specific antigens, it produces inhibitory cytokines to escape the host's immune defense (42). Moorhead et al. reported that $\mathrm{CD} 4^{+} \mathrm{T}$ cytokines in mice can be divided into two subgroups. Th1 type cells mainly secrete IL- 1 , IFN- $\gamma$, and TNF- $\beta$, and Th 2 cells mainly secrete IL-4, IL5 , and IL-10 (43). A previous study found that in patients with echinococcosis, Th2 type cytokines were mainly increased in their serum (44). Another study showed that the Th1-mediated cellular immune response can enhance the host's anti-infection ability in the early stage of echinococcosis, playing an important role in the control of the initial infection of echinococcosis (45). In the later stages of infection, the main humoral immune response is mediated by Th2 type immunity (45). It has also been suggested that the transformation from a Th1 immune response to a Th2 type immune response is beneficial for the growth and development of E. granulosus, such that IL-4, IL-5, IFN- $\gamma$, and other cytokines play an important role in the immune interaction between the host and E. granulosus (45). In the present study, rEg-TSP11 induced Th1 and Th2 immune responses in dogs, and the changes in IFN- $\gamma$ cytokine levels were more obvious in the pre-immune period. However, after challenge with PSCs for 28 days, marked changes in IL-5 cytokine levels were observed. Such delayed cytokine responses to PSC Ags have been previously described in infected mice and dogs $(20,46)$, which indicated that the humoral immune response mediated by Th2 type immunity is the main pathway in the late stage of echinococcosis.

We vaccinated dogs with Eg-TSP11 mixed with Quil A, and used PBS as a control. In terms of worm reduction rates, the rEgTSP11 immunized group showed a worm reduction of $76.80 \%$
TABLE 2 | Worm segment development in the experimental groups.

\begin{tabular}{lcccc}
\hline Group (protein) & Dog ID & \multicolumn{3}{c}{ Worm development } \\
\cline { 3 - 5 } & & $\leq 3$ segments & $\mathbf{2}$ s segments & Worm inhibition \\
\hline Eg-TSP11 & 1 & 26 & 4 & \\
& 2 & 22 & 8 & \\
Average & 3 & 28 & 2 & \\
PBS & & 25 & 5 & - \\
& 4 & 9 & 21 & \\
Average & 5 & 13 & 17 & \\
Quil A & 6 & 7 & 23 & \\
& & 9 & 21 & - \\
Average & 7 & 7 & 23 & \\
\hline
\end{tabular}

compared with that in the PBS control group, representing a significant reduction in the worm burden. Moreover, the rEgTSP11 immunized group showed a development and maturation inhibition of the worms was $77.04 \%$. However, 70.00 and $76.67 \%$ of worms developed to the adult worms in the PBS control groups and Quil A, while only $22.96 \%$ of worms developed to the adult worms in the rEg-TSP11 immunized group. This results also suggested that the $\mathrm{rEg}$-TSP11 vaccine induced significant protective efficacy in terms of inhibition of worm growth compared with the control dogs. This was better than the oral vaccine comprising a live carrier of Salmonella EgA31 EGTRP (70-80\%), although individual differences were large (21), and slightly lower than that of EgM123 (89.2\%) (19). Thus, rEg-TSP11 showed good performance in decreasing worm burden and inhibiting segment development. For humans, cattle, sheep, and other intermediate hosts, the threat comes from the eggs of adult E. granulosus. If a vaccine can be developed to reduce the number of parasites and inhibit their development by immunizing the definitive host, we might effectively reduce the amount of ovulation and reduce the threat. In this study, $\mathrm{rEg}$ TSP11 showed good performance in terms of the worm reduction rate and the inhibition of segmental development, and thus is expected to be a good vaccine candidate antigen for the definitive host of E. granulosus.

\section{CONCLUSIONS}

An increasing number of studies are focusing on the TSP because of its wide distribution and important biological roles in a variety of organisms. In the present study, we cloned and prokaryotically expressed Eg-TSP11. Furthermore, we evaluated the immunoprotective effects of rEg-TSP11 in model dogs. The results indicated that $\mathrm{rEg}$-TSP11 could induce the production of specific antibodies in dogs and significantly increase or decrease the levels of Th1 and Th2 cytokines. In addition, rEg-TSP11 decreased worm burden and inhibited segment development. 
These findings revealed that $\mathrm{rEg}$-TSP11 might be a potential candidate vaccine antigen against E. granulosus infection in dogs.

\section{DATA AVAILABILITY STATEMENT}

The original contributions presented in the study are included in the article/supplementary material, further inquiries can be directed to the corresponding author/s.

\section{ETHICS STATEMENT}

The animal study was reviewed and approved by the Care and Use of Laboratory Animals of the Xinjiang Academy of Agricultural and Reclamation Sciences (Shihezi, China) (Approval No. 2019-012, April 9, 2019). All animals were handled in strict accordance with the animal protection laws of the People's Republic of China (a draft animal protection law was released on September 18, 2009) and the National Standards for Laboratory Animals in China (executed on January 5, 2002).

\section{REFERENCES}

1. Eckert J, Deplazes P. Biological, epidemiological, and clinical aspects of echinococcosis, a zoonosis of increasing concern. Clin Microbiol Rev. (2004) 17:107-35. doi: 10.1128/CMR.17.1.107-135.2004

2. Atkinson JAM, Gray DJ, Clements ACA, Barnes TS, McManus DP, Yang YR. Environmental changes impacting Echinococcus transmission: research to support predictive surveillance and control. Glob Change Biol. (2013) 19:677-88. doi: 10.1111/gcb.12088

3. Wen H, Vuitton L, Tuxun T, Li J, Vuitton DA, Zhang W, et al. Echinococcosis: advances in the 21st Century. Clin Microbiol Rev. (2019) 32:e007518. doi: 10.1128/CMR.00075-18

4. Schweiger A, Ammann RW, Candinas D, Clavien PA, Eckert J, Gottstein B, et al. Human alveolar echinococcosis after fox population increase, Switzerland. Emerg Infect Dis. (2007) 13:878-82. doi: 10.3201/eid1306.061074

5. Torgerson PR, Keller K, Magnotta M, Ragland N. The global burden of alveolar echinococcosis. PLoS Negl Trop Dis. (2010) 4:e722. doi: 10.1371/journal.pntd.0000722

6. Budke CM, Carabin H, Ndimubanzi PC, Nguyen H, Rainwater E, Dickey M, et al. A systematic review of the literature on cystic echinococcosis frequency worldwide and its associated clinical manifestations. Am J Trop Med Hyg. (2013) 88:1011-27. doi: 10.4269/ajtmh.12-0692

7. Larrieu E, Mujica G, Araya D, Labanchi JL, Arezo M, Herrero E, et al. Pilot field trial of the EG95 vaccine against ovine cystic echinococcosis in Rio Negro, Argentina: 8 years of work. Acta Trop. (2019) 191:17. doi: 10.1016/j.actatropica.2018.12.025

8. Hu D, Song X, Xie Y, Zhong X, Wang N, Zheng Y, et al. Molecular insights into a tetraspanin in the hydatid tapeworm Echinococcus granulosus. Parasit Vect. (2015) 8:311. doi: 10.1186/s13071-015-0926-y

9. Levy S, Shoham T. The tetraspanin web modulates immune-signalling complexes. Nat Rev Immunol. (2005) 5:136-48. doi: 10.1038/nri1548

10. Tran MH, Freitas TC, Cooper L, Gaze S, Gatton ML, Jones MK, et al. Suppression of mRNAs encoding tegument tetraspanins from Schistosoma mansoni results in impaired tegument turnover. PLoS Pathog. (2010) 6:e1000840. doi: 10.1371/journal.ppat.1000840

11. Piratae S, Tesana S, Jones MK, Brindley PJ, Loukas A, Lovas E. et al. Molecular characterization of a tetraspanin from the human liver fluke, Opisthorchis viverrini. PLoS Negl Trop Dis. (2012) 6:e1939. doi: 10.1371/journal.pntd.0001939

\section{AUTHOR CONTRIBUTIONS}

JX, PZ, and NW performed the experiments, collected and analyzed the data, and prepared the manuscript. WW and $\mathrm{XM}$ contributed to study design and implementation. YZ and JM collected the parasite specimens and performed immunofluorescence experiments. ZW and $\mathrm{XB}$ conceived the study, participated in its design, coordinated the project, and contributed to the interpretation of the results. All authors contributed to the article and approved the submitted version.

\section{FUNDING}

This research was funded by the Important Science \& Technology Specific Projects of State Key Laboratory of Sheep Genetic Improvement and Healthy Production (grant number 2021ZD02), the National Natural Science Foundation of China (grant number 31860701), and the International Scientific and Technological Cooperation Projects of Xinjiang Production and Construction Corps (grant numbers 2021BC008 and 2020BC007).

12. Walker AJ. Insights into the functional biology of schistosomes. Parasit Vect. (2011) 4:203. doi: 10.1186/1756-3305-4-203

13. Cai $\mathrm{P}, \mathrm{Bu} \mathrm{L}$, Wang J, Wang Z, Zhong X, Wang H. Molecular characterization of Schistosoma japonicum tegument protein tetraspanin-2: sequence variation and possible implications for immune evasion. Biochem Biophys Res Commun. (2008) 372:197-202. doi: 10.1016/j.bbrc.2008.05.042

14. McWilliam HE, Driguez P, Piedrafita D, McManus DP, Meeusen EN. Discovery of novel Schistosoma japonicum antigens using a targeted protein microarray approach. Parasit Vect. (2014) 7:290. doi: 10.1186/1756-3305-7-290

15. Tran MH, Pearson MS, Bethony JM, Smyth DJ, Jones MK, Duke M, et al. Tetraspanins on the surface of Schistosoma mansoni are protective antigens against schistosomiasis. Nat Med. (2006) 12:835-40. doi: 10.1038/nm1430

16. Wang $\mathrm{H}$, Li J, Guo B, Zhao L, Zhang Z, McManus DP, et al. In vitro culture of Echinococcus multilocularis producing protoscoleces and mouse infection with the cultured vesicles. Parasit Vect. (2016) 9:411. doi: 10.1186/s13071-016-1687-y

17. Tamura K, Peterson D, Peterson N, Stecher G, Nei M, Kumar S. MEGA5: molecular evolutionary genetics analysis using maximum likelihood, evolutionary distance, and maximum parsimony methods. Mol Biol Evol. (2011) 28:2731-9. doi: 10.1093/molbev/msr121

18. Livak KJ, Schmittgen TD. Analysis of relative gene expression data using realtime quantitative PCR and the 2(-Delta Delta C(T)) Method. Methods. (2001) 25:402-8. doi: 10.1006/meth.2001.1262

19. Zhang ZZ, Guo G, Li J, Shi BX, Zhao L, Guo BP, et al. Dog vaccination with EgM proteins against Echinococcus granulosus. Infect Dis Poverty. (2018) 7:61. doi: 10.1186/s40249-018-0425-4

20. Pourseif MM, Moghaddam G, Nematollahi A, Khordadmehr M, Naghili B, Dehghani J, et al. Vaccination with rEGVac elicits immunoprotection against different stages of Echinococcus granulosus life cycle: a pilot study. Acta Trop. (2021) 218:105883. doi: 10.1016/j.actatropica.2021.105883

21. Petavy AF, Hormaeche C, Lahmar S, Ouhelli H, Chabalgoity A, Marchal T, et al. An oral recombinant vaccine in dogs against Echinococcus granulosus, the causative agent of human hydatid disease: a pilot study. PLoS Negl Trop Dis. (2008) 2:e125. doi: 10.1371/journal.pntd.0000125

22. Huang S, Yuan S, Dong M, Su J, Yu C, Shen Y, et al. The phylogenetic analysis of tetraspanins projects the evolution of cell-cell interactions from unicellular to multicellular organisms. Genomics. (2005) 86:67484. doi: 10.1016/j.ygeno.2005.08.004 
23. Szöllósi J, Horejsí V, Bene L, Angelisová P, Damjanovich S. Supramolecular complexes of MHC class I, MHC class II, CD20, and tetraspan molecules(CD53, CD81, and CD82) at the surface of a B cell line JY. $J$ Immunol. (1996) 157:2939-46.

24. Kropshofer H, Spindeldreher S, Röhn TA, Platania N, Grygar C, Daniel N, et al. Tetraspan microdomains distinct from lipid rafts enrich select peptideMHC class II complexes. Nat Immunol. (2002) 3:61-8. doi: 10.1038/ni750

25. Zhang W, Li J, Duke M, Jones MK, Kuang L, Zhang J, et al. Inconsistent protective efficacy and marked polymorphism limits the value of Schistosoma japonicum tetraspanin-2 as a vaccine target. PLoS Negl Trop Dis. (2011) 5:e1166. doi: 10.1371/journal.pntd.0001166

26. Cardoso FC, Macedo GC, Gava E, Kitten GT, Mati VL, de Melo AL, et al. Schistosoma mansoni tegument protein $\mathrm{Sm} 29$ is able to induce a Th1-type of immune response and protection against parasite infection. PLoS Negl Trop Dis. (2008) 2:e308. doi: 10.1371/journal.pntd.0000308

27. Jiang $\mathrm{N}$, Cai P, Yin J, Hao L, Lu H, Wang X, et al. Characterization of antibody responses to the Sj23 antigen of Schistosoma japonicum after infection and immunization. Acta Trop. (2010) 116:9-14. doi: 10.1016/j.actatropica.2010.04.015

28. Kim TY, Chung EJ, Sohn WM, Hong SH, Yong TS. Molecular characterization of Clonorchis sinensis tetraspanin 2 extracellular loop 2. Parasitol Res. (2012) 110:707-11. doi: 10.1007/s00436-011-2546-6

29. Dang Z, Yagi K, Oku Y, Kouguchi $H$, Kajino $K$, Watanabe J, et al. Evaluation of Echinococcus multilocularis tetraspanins as vaccine candidates against primary alveolar echinococcosis. Vaccine. (2009) 27:733945. doi: 10.1016/j.vaccine.2009.09.045

30. Dang Z, Yagi K, Oku Y, Kouguchi H, Kajino K, Matsumoto J, et al. A pilot study on developing mucosal vaccine against alveolar echinococcosis (AE) using recombinant tetraspanin 3: vaccine efficacy and immunology. PLoS Negl Trop Dis. (2012) 6:e1570. doi: 10.1371/journal.pntd.0001570

31. Dakshinamoorthy G, Munirathinam G, Stoicescu K, Reddy MV, Kalyanasundaram R. Large extracellular loop of tetraspanin as a potential vaccine candidate for filariasis. PLoS ONE. (2013) 8:e77394. doi: 10.1371/journal.pone.0077394

32. Lin RY, Wang JH, Lu XM, Zhou XT, Mantion G, Wen H, et al. Components of the mitogen-activated protein kinase cascade are activated in hepatic cells by Echinococcus multilocularis metacestode. World J Gastroenterol. (2009) 15:2116. doi: 10.3748/wjg.15.2116

33. Hopp TP, Woods KR. Prediction of protein antigenic determinants from amino acid sequences. Proc Natl Acad Sci USA. (1981) 78:38248. doi: 10.1073/pnas.78.6.3824

34. Wang $\mathrm{F}, \mathrm{Ye} \mathrm{B}$. In silico cloning and $\mathrm{B} / \mathrm{T}$ cell epitope prediction of triosephosphate isomerase from Echinococcus granulosus. Parasitol Res. (2016) 115:3991-8. doi: 10.1007/s00436-016-5 166-3

35. Avril C, Rahul T, James AC, Nancy H, Bruce AR, Isheng JT, et al. Comparative genomics of the major parasitic worms. Nat Genet. (2019) 51:163-74. doi: 10.1038/s41588-018-0 262-1

36. Coakley G, Maizels RM, Buck AH. Exosomes and other extracellular vesicles: the new communicators in parasite infections. Trends Parasitol. (2015) 31:477-89. doi: 10.1016/j.pt.2015.06.009
37. Wu C, Cai PF, Chang QC, Hao LL, Peng S, Sun XJ, et al. Mapping the binding between the tetraspanin molecule (Sjc23) of Schistosoma japonicum and human non-immune IgG. PLoS ONE. (2011) 6:e19112. doi: 10.1371/journal.pone.0019112

38. Krautz-Peterson G, Debatis M, Tremblay JM, Oliveira SC, Da'dara AA, Skelly PJ, et al. Schistosoma mansoni infection of mice, rats and humans elicits a strong antibody response to a limited number of reduction-sensitive epitopes on five major tegumental membrane proteins. PLoS Negl Trop Dis. (2017) 11:e0005306. doi: 10.1371/journal.pntd.0005306

39. Reed SG, Orr MT, Fox CB. Key roles of adjuvants in modern vaccines. Nat Med. (2013) 19:1597-608. doi: 10.1038/nm.3409

40. Singh M, O'Hagan DT. Recent advances in veterinary vaccine adjuvants. Int $J$ Parasitol. (2003) 33:469-78. doi: 10.1016/S0020-7519(03)00053-5

41. Sun HX, Xie Y, Ye YP. ISCOMs and ISOMATRIX. Vaccine. (2009) 27:4388401. doi: 10.1016/j.vaccine.2009.05.032

42. Ortona E, Margutti P, Delunardo F, Nobili V, Profumo E, Riganò R, et al. Screening of an Echinococcus granulosus cDNA library with IgG4 from patients with cystic echinococcosis identifies a new tegumental protein involved in the immune escape. Clin Exp Immunol. (2005) 142:52838. doi: 10.1111/j.1365-2249.2005.02939.x

43. Moorhead JF, Chan MK, El-Nahas M, Varghese Z. Lipid nephrotoxicity in chronic progressive glomerular and tubulo-interstitial disease. Lancet. (1982) 320:1309-11. doi: 10.1016/S0140-6736(82)91513-6

44. Newport MJ, Finan C. Genome-wide association studies and susceptibility to infectious diseases. Brief Funct Genomics. (2011) 10:98. doi: 10.1093/bfgp/elq037

45. Mourglia-Ettlin G, Marqués JM, Chabalgoity JA, Dematteis S. Early peritoneal immune response during Echinococcus granulosus establishment displays a biphasic behavior. PLoS Negl Trop Dis. (2011) 5:e1293. doi: 10.1371/journal.pntd.0001293

46. Rostami-Rad S, Jafari R, Yousofi Darani H. Th1/Th2-type cytokine profile in C57 black mice inoculated with live Echinococcus granulosus protoscolices. J Infect Public Health. (2018) 11:834-9. doi: 10.1016/j.jiph.2018.06.007

Conflict of Interest: The authors declare that the research was conducted in the absence of any commercial or financial relationships that could be construed as a potential conflict of interest.

Publisher's Note: All claims expressed in this article are solely those of the authors and do not necessarily represent those of their affiliated organizations, or those of the publisher, the editors and the reviewers. Any product that may be evaluated in this article, or claim that may be made by its manufacturer, is not guaranteed or endorsed by the publisher.

Copyright (c) 2021 Xian, Zhao, Wang, Wang, Zhang, Meng, Ma, Wang and Bo. This is an open-access article distributed under the terms of the Creative Commons Attribution License (CC BY). The use, distribution or reproduction in other forums is permitted, provided the original author(s) and the copyright owner(s) are credited and that the original publication in this journal is cited, in accordance with accepted academic practice. No use, distribution or reproduction is permitted which does not comply with these terms. 